\title{
A mathematical model with control to analyse the dynamics of dengue disease transmission in urban Colombo
}

\author{
W.P.T.M. Wickramaarachchi ${ }^{1 *}$ and S.S.N. Perera ${ }^{2}$ \\ ${ }^{1}$ Department of Mathematics, The Open University of Sri Lanka, Nawala, Nugegoda. \\ ${ }^{2}$ Research and Development Centre for Mathematical Modelling, Faculty of Science, University of Colombo, Colombo 03.
}

Revised: 19 July 2017; Accepted: 17 August 2017

\begin{abstract}
Dengue is a mosquito-borne disease. It has been an important public health problem particularly in the tropical and sub-tropical regions in the world, for which no vaccine or successful treatment has been found. Therefore, prevention and control play a vital role in minimising the risk of dengue in vulnerable populations.
\end{abstract}

Various mathematical models such as the SIR model have been developed to understand the transmission dynamics of dengue. The main drawback of these dynamic models is the lack of predictability with respect to external factors such as climate, geography, demography and human behaviour due to usage of fixed parameters. The mosquito density, which depends heavily on various external factors such as climate is a critical parameter of these models and is responsible for the local transmission of the disease. In this study, the mosquito density is modelled with respect to changing levels of climate favourable for mosquito reproduction. A climate risk index developed using fuzzy set theory is used to vary the mosquito density with respect to rainfall and temperature and this measure is included in the SIR model.

Finally, two measures, $u_{1}$ and $u_{2}$, are introduced to control adult mosquitoes and growing juveniles using two methods. The first method is theoretical where we consider $u_{1}$ and $u_{2}$ as random processes from uniform distribution. The second method involves continuous and constant control measures over time. The numerical results of the SIR model suggest that the dynamics of infections has changed and the number of infections is reduced as the efficiency of the control measures increase.

Keywords: Control, dengue, fuzzy set theory, mathematical models.

\section{INTRODUCTION}

Dengue fever (DF) and its severe form, dengue haemorrhagic fever (DHF) are the most common and widespread vector borne diseases in the world. According to the World Health Organization (WHO), dengue disease is ranked as one of the most critical infectious diseases with severe impact on public health and well-being of the society (Racloz et al., 2012; Thai, 2012). The disease has spread to almost all the tropical and sub-tropical parts in the world and it has been estimated that nearly 2.5 billion people in more than 100 countries are at risk (Bhatia et al., 2013). Globally, every year, approximately 50 million dengue infections occur; and half a million DHF cases require hospitalisation with over 20,000 deaths (Thai, 2012; Bhatia et al., 2013; Murray et al., 2013). The economic impact of DF/DHF is massive, placing significant burdens on affected nations and their communities. This impact varies and can include deaths, medical expenditure for hospitalisation of patients and their careful clinical management, loss in productivity of the affected workforce, strain on healthcare services due to sudden, high demand during an epidemic, considerable expenditures for large-scale emergency control actions taken by the government in an outbreak etc. (Lloyd, 2003).

DF and DHF are endemic in Sri Lanka now since the first reported outbreak of DF in 1965 (Epidemiology Unit, Ministry of Health, 2005). There has been recurring outbreaks for the last five decades creating severe damages

\footnotetext{
*Corresponding author (wptharindu86uoc@gmail.com; (iD https://orcid.org/0000-0002-6377-7859)
} 
to public health and the well-being of Sri Lankan people. According to the Epidemiology unit, Ministry of Health, Sri Lanka (http://www.epid.gov.lk/web), during the first half of the year 2016, there were nearly 21,500 dengue cases reported in Sri Lanka with a notifiable number of deaths. Of them, the majority have been from Colombo. Colombo is the capital and the largest city in Sri Lanka; its rapid unsystematic urbanisation and increased human movements have resulted in Colombo becoming a highly vulnerable geographic area for dengue disease (Thalagala, 2012).

There is no vaccine or any specific treatment found for dengue disease and several researches for a vaccine are currently in progress (Parks \& Lloyd, 2004; Bhatia et al., 2013). For the time being, the only mechanism for preventing and controlling DF/DHF is to ensure prompt diagnosis of cases of fever and appropriate clinical management during the hospitalisation, to reduce humanvector contact, and to control larval habitats (Parks \& Lloyd, 2004).

Dengue is a vector borne disease with a complex transmission process. Dengue transmission dynamics are influenced by many factors, such as dynamical behaviour patterns of humans and mosquitoes, and the degree of interaction between humans versus mosquitoes. Further, both humans and mosquitoes interact heavily with the environment as well. The variation in climate factors such as rainfall, temperature and humidity are also influential to dengue disease transmission. Temperature and rainfall interfere at all stages of mosquito development from the emergence and viability of eggs, to the size and longevity of adult mosquitoes, as well as their dispersal over space (Allis et al., 2011; Medeiros et al., 2011). Demographic factors such as population density, education level, human awareness and household income are also vital determinants of dengue spread. Human mobility creates a large number of human contacts and this may speed up the dengue disease transmission (Allis et al., 2011).

Exploring the complexity of infectious disease systems such as dengue disease and understanding its transmission dynamics is important to identify control measures effectively. Mathematical models of dengue transmission reveal useful information about its complex dynamic nature and the information can be applied to control the spread of the disease and minimise the disease burden.

This paper focuses mainly on the importance of controlling dengue mosquitoes at two stages of their life cycle, namely, growing juveniles and adult mosquitoes.
We consider the SIR model for dengue, which describes the transmission of dengue disease in terms of population dynamics. The human population is divided into three compartments, namely, susceptible (S), infected (I) and recovered $(\mathrm{R})$ while the mosquito population is divided into two compartments, namely, susceptible and infected (Estevaa \& Vargas, 1998; Pongsumpun, 2006; Ma \& Xia, 2009). The mosquito density is a critical parameter in these mathematical models since it is the driving factor for the local transmission of dengue and it depends heavily on various external factors, particularly climate. We model this parameter using a discrete nonlinear dynamic model. The climate effect on mosquitoes is modelled using fuzzy set theory. The constructed fuzzy measure describes the level of favourability to dengue mosquitoes from climate. This risk measure is also included in the discrete non-linear dynamic model for mosquitoes.

We introduce two measures $u_{1}$ and $u_{2}$ to control adult mosquitoes and growing juveniles and implement control measures with several combinations of $u_{1}$ and $u_{2}$. First we consider the two control measures as uniformly distributed random variables. Next we consider that the two measures are constants and they are continuously implemented over time. Finally the entire system is numerically solved to investigate the consequences of these control strategies. The fourth order Runge Kutta scheme is used to obtain the solutions (Cartwright \& Piro, 1992; Suli, 2014)

\section{METHODOLOGY}

\section{Fuzzy set theory}

Definition 1 A fuzzy subset $F$ of $U$ is described by the function $F: U \rightarrow[0,1]$ called the membership function of fuzzy set $F$ where $U$ is a classical non-empty set. The value $F(x) \in[0,1]$ indicates the membership degree of the element $x$ of $U$ in fuzzy set $F$, with $F(x)=1$ and $F(x)=0$ representing, respectively, the belongingness and non-belongingness of $x$ in $F$.

Definition 2 The intersection of $A$ and $B$, denoted $A \cap B$, is defined on the largest fuzzy set contained in both $A$ and $B$, given by the membership function $U_{A \cap B}(x)=\min \left\{U_{A}(x), U_{B}(x)\right\}$ for each $x \in F$. The union of $A$ and $B$, denoted $A \cup B$, is defined on the largest fuzzy set contained in both $A$ and $B$, given by the membership function $U_{A \cup B}(x)=\max \left\{U_{A}(x), U_{B}(x)\right\}$ for each $x \in F$. The Einstein sum is defined as $U_{E S}(x)=\frac{U_{A}(x)+U_{B}(x)}{1+U_{A}(x) \cdot U_{B}(x)}($ Zimmermann, 2010). 


\section{Mathematical model for dengue transmission}

We consider the mathematical model for dengue transmission described by a system of non-linear ordinary differential equations (Estevaa \& Vargas, 1998; Pongsumpun, 2006). This model describes the interaction between susceptible, infected and recovered human populations together with susceptible and infected mosquito populations.

$$
\begin{aligned}
\frac{d S^{h}}{d t} & =\lambda N_{T}-\frac{b \beta_{h}}{N_{T}} S^{h} I^{v}-\mu_{h} S^{h} \\
\frac{d I^{h}}{d t} & =\frac{b \beta_{h}}{N_{T}} S^{h} I^{v}-\left(\mu_{h}+r\right) I^{h} \\
\frac{d R^{h}}{d t} & =r I^{h}-\mu_{h} R^{h} \\
\frac{d S^{v}}{d t} & =D-\frac{b \beta_{v}}{N_{T}} S^{v} I^{h}-\mu_{v} S^{v} \\
\frac{d I^{v}}{d t} & =\frac{b \beta_{v}}{N_{T}} S^{v} I^{h}-\mu_{v} I^{v}
\end{aligned}
$$

with $N_{T}=S^{h}+I^{h}+R^{h}$ and $N_{v}=S^{v}+I^{v}$

where

$N_{T}$ - the total number of human population

$\lambda$ - the birth rate of the human population

$b$ - the biting rate of the mosquitoes

$\beta_{h}-$ the transmission probability of the virus from mosquitoes to humans

$\beta_{v}-$ the transmission probability of the virus from humans to mosquitoes

$\mu_{h}-$ the death rate of the human population

$r$ - the recovery rate of the human population

$D$ - the constant recruitment rate of the mosquito population

$\mu_{v}$ - the death rate of the mosquito population

and

$S^{h}$ - susceptible human population

$I^{h}$ - infected human population

$R^{h}$ - recovered human population

$S^{v}$ - susceptible mosquito population

$I^{v}$ - infected mosquito population

$N_{T}-$ total human population

$N_{v}-$ total mosquito population.

Suppose the mosquito population is a constant in time. This gives us $\frac{d N_{v}}{d t}=0$. Since $N_{v}=S^{v}+I^{v}$, we have $\frac{d N_{v}}{d t}=\frac{d S^{v}}{d t}+\frac{d I^{v}}{d t}$. These two conditions and the last two equations in system (1) gives $N_{v}=\frac{D}{\mu_{v}}$.
Now we assume that the mosquito population $N_{v}$ changes in time (is nota constant) that is $\frac{d N_{v}}{d t}=q(t)$ where $q(t) \neq 0$. However, we assume that the total human population is a constant hence $\frac{d N_{T}}{d t}=0$. Then we obtain $\lambda=\mu_{h}$ and the mosquito population as $N_{v}=\frac{D-q}{\mu_{v}}$. Now we define the proportion $n=\frac{N_{v}}{N_{T}}$ as the number of mosquitoes per human. We let, $S=\frac{S^{h}}{N_{T}}, I=\frac{I^{h}}{N_{T}}, R=\frac{R^{h}}{N_{T}}, S_{v}=\frac{S^{v}}{N_{v}}$ and $I_{v}=\frac{I^{v}}{N_{v}}$ and the system in dimensionless form, reduced to the susceptible human, infected human, susceptible mosquito and infected mosquito populations given by

$$
\begin{aligned}
& \frac{d S}{d t}=\lambda-b \beta_{h} n(t) S I_{v}-\mu_{h} S \\
& \frac{d I}{d t}=b \beta_{h} n(t) S I_{v}-\left(\mu_{h}+r\right) I \\
& \frac{d S_{v}}{d t}=\frac{D \mu_{v}}{D-q}-b \beta_{v} S_{v} I-\mu_{v} S_{v} \\
& \frac{d I_{v}}{d t}=b \beta_{v} S_{v} I-\mu_{v} I_{v}
\end{aligned}
$$

where $n(t)=\frac{N_{v}}{N_{T}}=\frac{D-q / \mu_{v}}{N_{T}}$. Since $N_{v}$ depends on time, $n$ can be modelled as a function of time $t$ defined as $n(t)$.

Although $N_{v}$ depends on time $t$, it should be noted that $S_{v}(t)+I_{v}(t)=\frac{S^{v}(t)}{N_{v}(t)}+\frac{I^{v}(t)}{N_{v}}=\frac{1}{N_{v}(t)} \cdot N_{v}(t)=1$ for any given time $t$. Therefore, system (3) can be reduced to

$$
\begin{aligned}
& \frac{d S}{d t}=\lambda-\gamma_{h} n(t) S I_{v}-\mu_{h} S \\
& \frac{d I}{d t}=\gamma_{h} n(t) S I_{v}-\left(\mu_{h}+r\right) I \\
& \frac{d I_{v}}{d t}=\gamma_{v}\left(1-I_{v}\right) I-\mu_{v} I_{v},
\end{aligned}
$$

where $\gamma_{h}=b \beta_{h}$ and $\gamma_{v}=b \beta_{v}$ with $S(0)=S_{0}>0, I(0)=I_{0}>0$. and $I_{v}(0)=I_{v 0}>0$. This model is an improved model where $n(t)$ captures the periodic variation of the external forces for mosquitoes.

\section{Model for mosquito density}

The development of the discrete non-linear model for mosquito reproduction is discussed here. 


\section{Gompertz model}

The Gompertz model is defined as (Turchin, 2003)

$N(t+1)=\lambda_{0} N(t)^{\theta}$

where $\theta \in[0,1]$ is an exponent, $\lambda_{0}=\exp \left(r_{0}\right), N(t)$ is the size of the population at time $t$ and $r_{0}$ is the population growth rate.

Let us start with a simple model in which we suppose that the number of mosquitoes at time $t,\left(N_{v}(t)\right)$ depends on the adult mosquitoes at time $t-1,\left(N_{v}(t-1)\right)$ and the number of growing juvenile mosquitoes at time $t-1$, $(J(t-1))$ (Franke \& Yakubu, 2005; Chaves et al., 2011). This model can now be expressed as

$N_{v}(t)=s N_{v}(t-1)+p J(t-1)$

where $s$ is the per capita survival rate of the adult mosquitoes and $p$ is the per capita growing probability of juveniles.

The number of juveniles at time $t,(J(t))$ is regulated by the adult mosquito density in the previous time step. Thus, we can write

$J(t)=F\left(N_{v}(t-1)\right)$

Using Gompertz model in equation (5) and we write $J(t)=F(N(t-1))$ be defined as

$J(t)=\lambda_{0} N_{v}^{\theta}(t)$.

Now equation (6) can be expressed as

$N_{v}(t)=s N_{v}(t-1)+p\left(\lambda_{0} N_{v}^{\theta}(t-2)\right)$.

\section{Modelling the climate force}

It is identified that at least $5 \mathrm{~mm}$ averaged weekly rainfall is required to make breeding sites available for mosquitoes and the breeding sites are washed out due to the heavy rainfall, which is over $55 \mathrm{~mm}$ (Huang et al., 2013; Naish et al., 2014). Further it is identified that a weekly average temperature less than $16{ }^{\circ} \mathrm{C}$ is unfavourable for mosquitoes to transmit the virus and a temperature between $30{ }^{\circ} \mathrm{C}$ and $34{ }^{\circ} \mathrm{C}$ is ideal for mosquitoes to survive. It is noted that extreme warm conditions do not support dengue mosquitoes, so that we assumed our threshold temperature to be $37{ }^{\circ} \mathrm{C}$ (Huang et al., 2013; Naish et al., 2014). Based on these conditions, we defined the trapezoidal-shaped membership functions $U_{R F}(x): A \subseteq R \rightarrow[0,1]$ and $U_{\text {TEMP }}(x): B \subseteq R \rightarrow[0,1]$ respectively to represent the effect from eight weeks leading rainfall (RF), and immediate temperature (TEMP) to create an unfavourable environment for dengue mosquitoes as

$U_{R F}(x)= \begin{cases}1 & \text { if } x \leq 5 \\ -\frac{x+10}{5} & \text { if } 5 \leq x \leq 10 \\ 0 & \text { if } 10 \leq x \leq 30 \\ \frac{x-30}{25} & \text { if } 30 \leq x \leq 55 \\ 1 & \text { if } x \geq 55\end{cases}$

$U_{\text {TEMP }}(x)= \begin{cases}1 & \text { if } x \leq 16 \\ -\frac{x+30}{14} & \text { if } 16 \leq x \leq 30 \\ 0 & \text { if } 30 \leq x \leq 34 \\ \frac{x-34}{3} & \text { if } 34 \leq x \leq 37 \\ 1 & \text { if } x \geq 37 .\end{cases}$

The trapezoidal-shaped membership functions given in equations (10) and (11) are illustrated in Figure 1.

\section{Fuzzy operator}

The modified operator, which computes the overall effect as a function is defined as $U_{M E S}(x):([0,1] \times[0,1]) \rightarrow[0,1]$ and it is given by

$U_{M E S}(x)=\frac{U_{R F}^{2}(x)+U_{T E M P}^{2}(x)}{1+U_{R F}(x) \cdot U_{T E M P}(x)}$.

The behaviour of the overall effect $U_{M E S}(x)$ for different membership values of RF and TEMP is represented in Figure 2.

The measure of climate favourability on dengue mosquitoes is now defined as $M:([0,1] \times[0,1]) \rightarrow[0,1]$ and given by

$M(t)=1-U_{M E S}(t)$.

Here, $t$ is the time.

This fuzzy measure of climate favourability is additively included into ourmodel and it is assumed that the adult mosquitoes in time $t-1, N_{v}(t-1)$ take the advantage of this climate favourability. Now $N_{v}(t)$ can be viewed as a summation of two components $F_{1}(t)$ and $F_{2}(t)$ where 

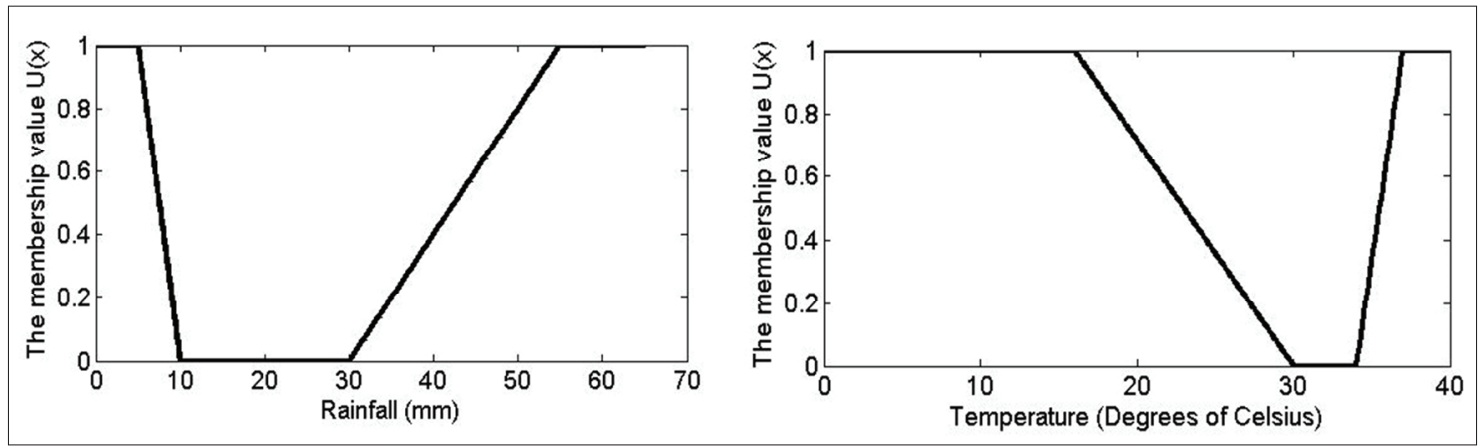

Figure 1: Membership functions for eight weeks leading average weekly rainfall (left) and the average weekly temperature (right)

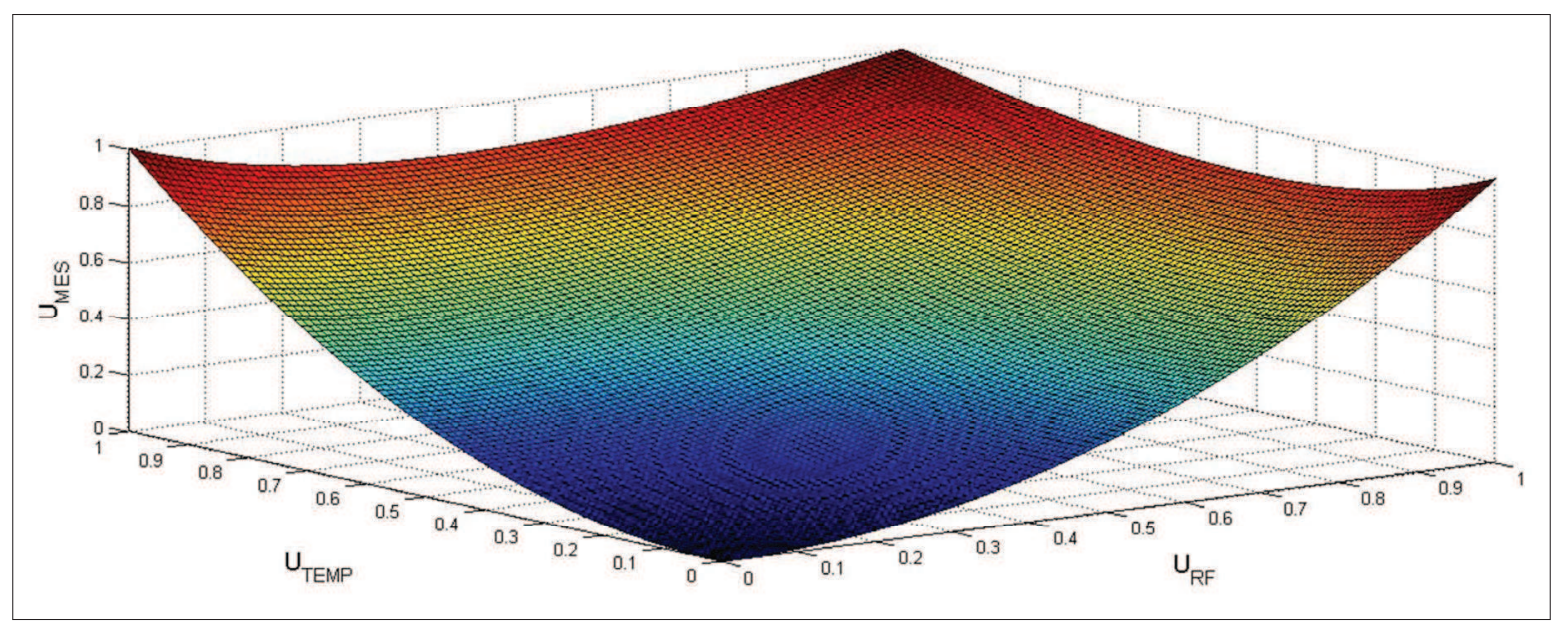

Figure 2: The behaviour of the overall effect for different membership values of RF and TEMP

$F_{1}(t)=s N_{v}(t-1)+p\left(\lambda_{0} N_{v}^{\theta}(t-2)\right)$ which depends on biological factors and $F_{2}(t)=F(R F, T E M P)$. Finally, we can write our fuzzy discrete-time dengue mosquito density model as

$N_{v}(t)=s N_{v}(t-1)+p\left(\lambda_{0} N_{v}^{\theta}(t-2)\right)+c M(t-1) N_{v}(t-1)$

where $c \in[0,1]$ is the coefficient of climate force and $\theta$ is defined as in equation (5). It should be noted that if $c=0$ then the climate does not have any influence to mosquito reproduction and if $c=1$ then the climate fully acts on mosquitoes. We define the mosquito density as $n(t)=\frac{N_{v}(t)}{N_{T}}$ with the same notations in system (4). Now we express our mosquito density model as

$$
n(t)=\operatorname{sn}(t-1)+p\left(\lambda_{0} n^{\theta}(t-2)\right)+c M(t-1) n(t-1)
$$

(It is assumed that the mosquito lifetime is negligible compared to the lifetime of a human and $\left.n^{\theta}(t) \approx \frac{N_{v}^{\theta}(t)}{N_{T}}\right)$.

\section{Mathematical model with control measures}

It should be noted that there is no control over the climate since it is a natural phenomenon, but the impact of climate conditions can be controlled. Therefore, the growth rate of mosquitoes and their survival rates can be controlled. Here, we investigate the effect of two control measures, namely $u_{1} \in[0,1]$ and $u_{2} \in[0,1]$ :

- Controlling the adult mosquitoes, which is referred to reducing the survivability of adult mosquitoes those who can survive to time $t$ from time $t-1$. The level of control is denoted by $u_{1} \in[0,1]$. Practically this control measure includes chemical methods directed 
against adult mosquitoes, such as insecticide space sprays or residual applications (Parks \& Lloyd, 2004).

- Controlling the growing juveniles, which is referred to reducing the growth of juveniles at time $t-1$ to become adult mosquitoes at time $t$. The level of control is denoted by $u_{2} \in[0,1]$. This control measure includes the biological methods [e.g. fish, copepods (small crustaceans) that feed on mosquito larvae] to kill or reduce larval mosquito populations in water containers and chemical methods against the mosquito aquatic stage for use in water containers (e.g. temephos sand granules) (Parks \& Lloyd, 2004).

With these two control measures $u_{1}$ and $u_{2}$, equation (15) can be expressed as

$n(t)=\left(1-u_{1}\right) \operatorname{sn}(t-1)+\left(1-u_{2}\right) p\left(\lambda_{0} n^{\theta}(t-2)\right)+c M(t-1) n(t-1)$

\section{Implementing control measures}

It should be noted that the scenario where $u_{1}=u_{2}=1$ is hardly achieved practically. The levels of these control measures are allowed to vary with respect to time (not fixed). The simulation of the model is carried out using two methods:

\section{Method I:}

The efficiency of the control activities taken place are uncertain. Therefore, we use a probabilistic approach to model this. Here we let $u_{1}$ and $u_{2}$ be uniformly distributed random variables in $\left[\bar{u}_{1}, \underline{u}_{1}\right] \subset[0,1]$ and $\left[\bar{u}_{2}, \underline{u}_{2}\right] \subset[0,1]$. We perform the simulation with four combinations of the two random variables as

i. $\quad u_{1} \sim$ uni $[0,0.4]$ and $u_{2} \sim[0,0.25]$

ii. $u_{1} \sim$ uni $[0.1,0.5]$ and $u_{2} \sim[0.1,0.3]$

iii. $u_{1} \sim \operatorname{uni}[0.2,0.6]$ and $u_{2} \sim[0.2,0.35]$

iv. $u_{1} \sim u n i[0.3,0.7]$ and $u_{2} \sim[0.3,0.45]$.

\section{Method II:}

It is observed that rainfall is the driving force for dengue outbreaks in urban Colombo due to the increased level of dengue mosquitoes during the rainy seasons. The temperature in Colombo does not vary significantly and it is favourable for dengue mosquitoes throughout the year. The control measures should be implemented to avoid the environmental favourability produced by this changing levels of rainfall. Thus it is expected to have a constant level of effective control activities from the public to eradicate dengue mosquitoes from the environment. Therefore, we let the effect from temperature is fixed over time at $29^{\circ} \mathrm{C}$ (as an average measure). Appropriate measures are then implemented to control the mosquito density and hence we expect to reduce the number of dengue infections in humans. We investigate the conditional distribution of infected humans given eight weeks leading rainfall and some level of control measures, which is defined as $f\left(I^{h} \mid R F, \underline{u}\right)$ where $\underline{u}=\left(u_{1}, u_{2}\right)$. Four combinations of the two control measures $u_{1}$ and $u_{2}$ are considered for the simulations. We assume that neither $u_{1}$ nor $u_{2}$ is achieved at $100 \%$ efficiency. The scenarios considered for the simulation are,

i. $\quad u_{1}=0$ and $u_{2}=0$

ii. $u_{1}=0.25$ and $u_{2}=0.10$

iii. $u_{1}=0.50$ and $u_{2}=0.30$

iv. $u_{1}=0.70$ and $u_{2}=0.50$.

\section{Algorithm}

1. Construction of fuzzy membership functions according to equations (10) and (11) for eight weeks leading rainfall and immediate temperature.

2. For $t=1: T$, generate fuzzy measures of favourability for dengue mosquitoes from rainfall and temperature.

3. For $t=1: T$, generate the fuzzy overall measure using modified Einstein sum operator in equation (12).

4. For $t=1: T$, generate the mosquito density $n(t)$ using equation (16) by introducing the two control measures $u_{1}$ and $u_{2}$.

5. For $t=1: T$, input $n(t)$ and solve the system (4) using Runge Kutta method of order 4.

\section{RESULTS AND DISCUSSION}

Figure 3 shows the simulated mosquito density dynamics without control measures according to equation (15) [Figure 3(a)] and the corresponding dynamics of the infected human population [Figure 3(b)]. The weekly average rainfall and temperature data in Colombo from year 2006 to 2011 is used for the simulation. The mosquito density shows a periodic variation in time and this is due to the varying levels of climate favourability. The infected human population is also periodic with some increasing trend.

The dynamics of the mosquito density with uniformly distributed two control measures are shown in Figure 4. The corresponding infected human population dynamics is represented in Figure 5. It can be seen that the distribution of infected human population has changed after introducing 


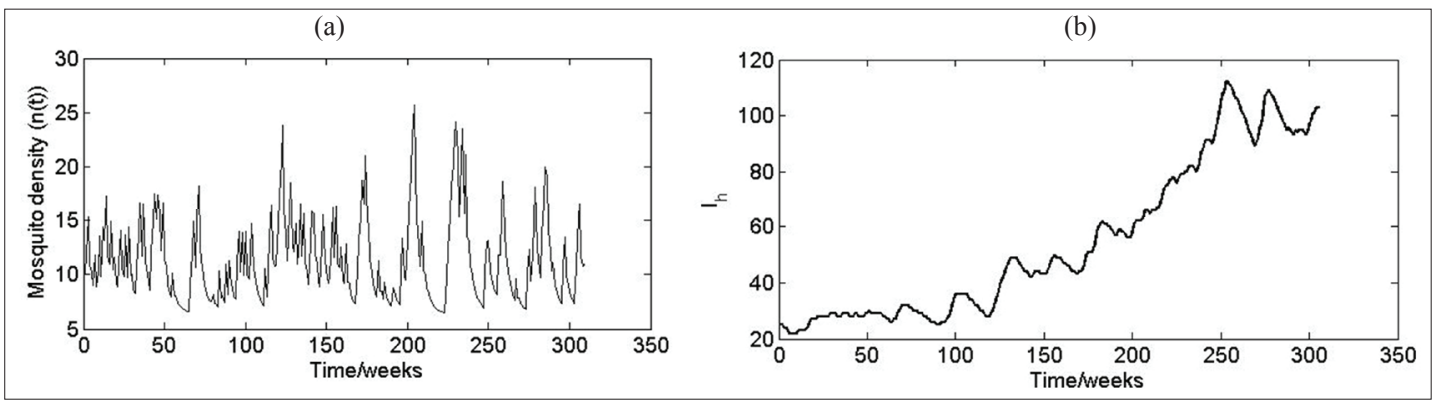

Figure 3: Mosquito density dynamics without control measures implemented (a) and the simulated infected human population distribution in Colombo from year 2006-2011 (b) with the parameter values $\lambda=\mu_{h}=0.0000391 ; \mu_{v}=0.071 ; \mathrm{r}=1 / 14$; $\beta_{h}=0.5 ; \beta_{v}=0.7$ and $b=1 / 3$. In equation (15), $s=0.317 ; P \lambda_{0}=1.71 ; c=0.5$ and $\theta=0.505$ (Chaves et al., 2011). The varying mosquito density is used without control according to the model in equation (15)

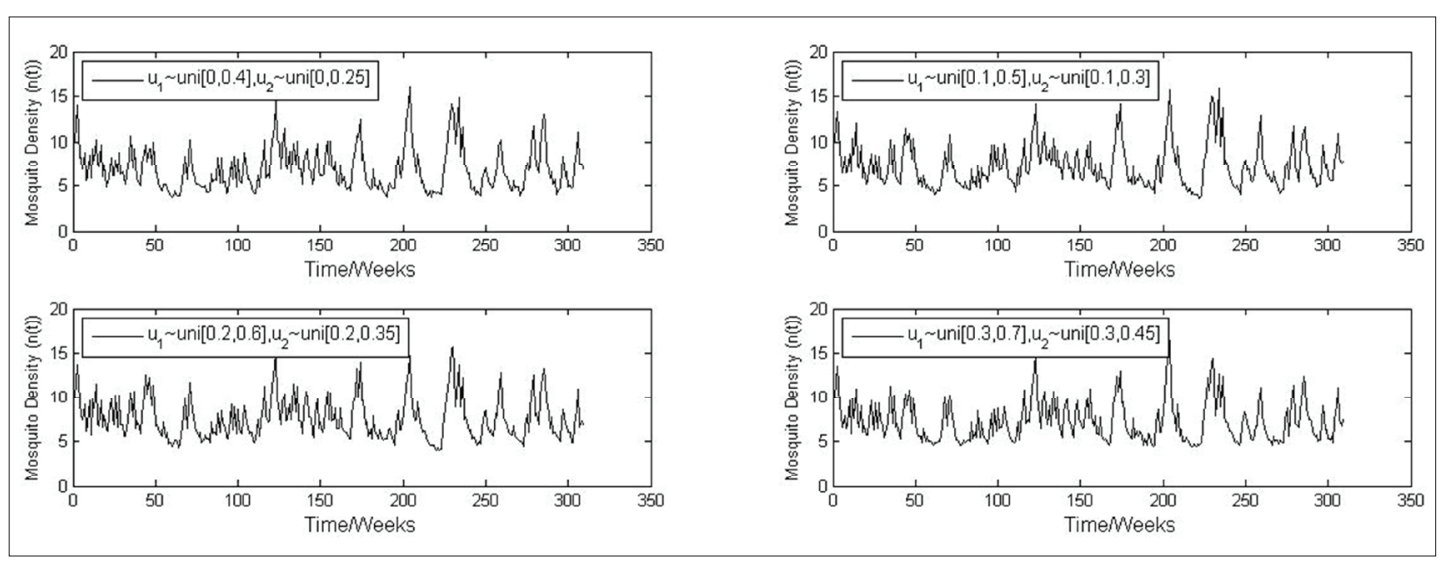

Figure 4: Simulated mosquito density dynamics in Colombo from year 2006 - 2011 with control measures theoretically implemented as $u_{1}(t)$ and $u_{2}(t)$ are uniformly distributed random variables. Here, $u_{1} \sim u n i[0,0.4]$ and $u_{2} \sim[0,0.25]$ (topleft); $u_{1} \sim$ uni $[0.1,0.5]$ and $u_{2} \sim[0.1,0.3]$ (top-right); $u_{1} \sim$ uni $[0.2,0.6]$ and $u_{2} \sim[0.2,0.35]$ (bottom-left); $u_{1} \sim u n i[0.3,0.7]$ and $u_{2} \sim[0.3,0.45]$ (bottom-right). In equation (16), $s=0.317 ; P \lambda_{0}=1.71 ; c=0.5$ and $\theta=0.505$ (Chaves et al., 2011)

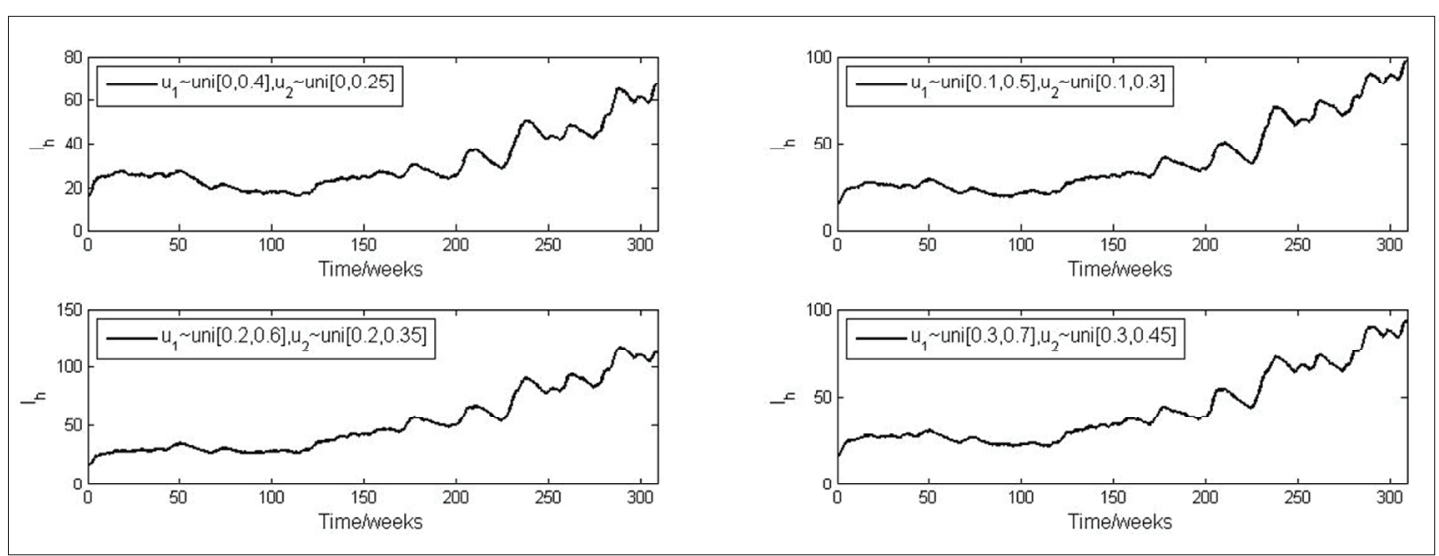

Figure 5: Simulated infected human population distribution in Colombo from year 2006 - 2011 with control measures theoretically implemented as $u_{1}(t)$ and $u_{2}(t)$ are uniformly distributed random variables. Here, $u_{1} \sim u n i[0,0.4]$ and $u_{2} \sim$ [0,0.25](top-left); $u_{1} \sim$ uni $[0.1,0.5]$ and $u_{2} \sim[0.1,0.3]$ (top-right); $u_{1} \sim u n i[0.2,0.6]$ and $u_{2} \sim[0.2,0.35]$ (bottom-left); $u_{1} \sim$ uni $[0.3,0.7]$ and $u_{2} \sim[0.3,0.45]$ (bottom-right) 
a small level of control, which varies randomly with respect to time. Figure 5 reveals theoretically that the randomly varying control measures reduce the number of infections compared to the dynamics of the infected human population in Figure 3 (right).
Figure 6 represents the dynamics of mosquito density with respect to different levels of control measures. It can be seen from Figure 6 that the simulated dengue mosquito density shows a reduction as the two control measures $u_{1}$ and $u_{2}$ are increased. This simulation indicates
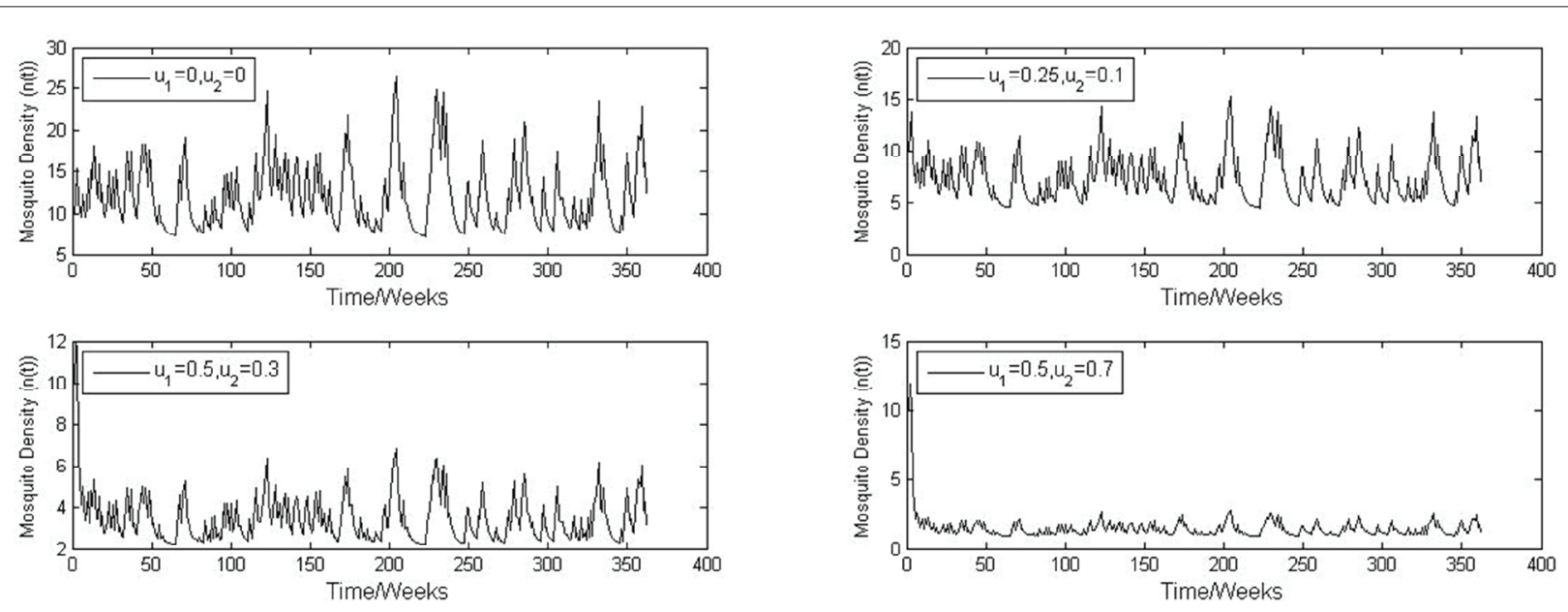

Figure 6: Simulated dengue mosquito density only with the rainfall variation (fixed temperature effect) for various combinations of constant continuous control measures from year $2006-2012$ in Colombo. Here, $u_{1}=0$ and $u_{2}=0$ (top-left); $u_{1}=0.25$ and $u_{2}=0.1$ (top-right); $u_{1}=0.5$ and $u_{2}=0.3$ (bottom-left); $u_{1}=0.5$ and $u_{2}=0.7$ (bottom-right)
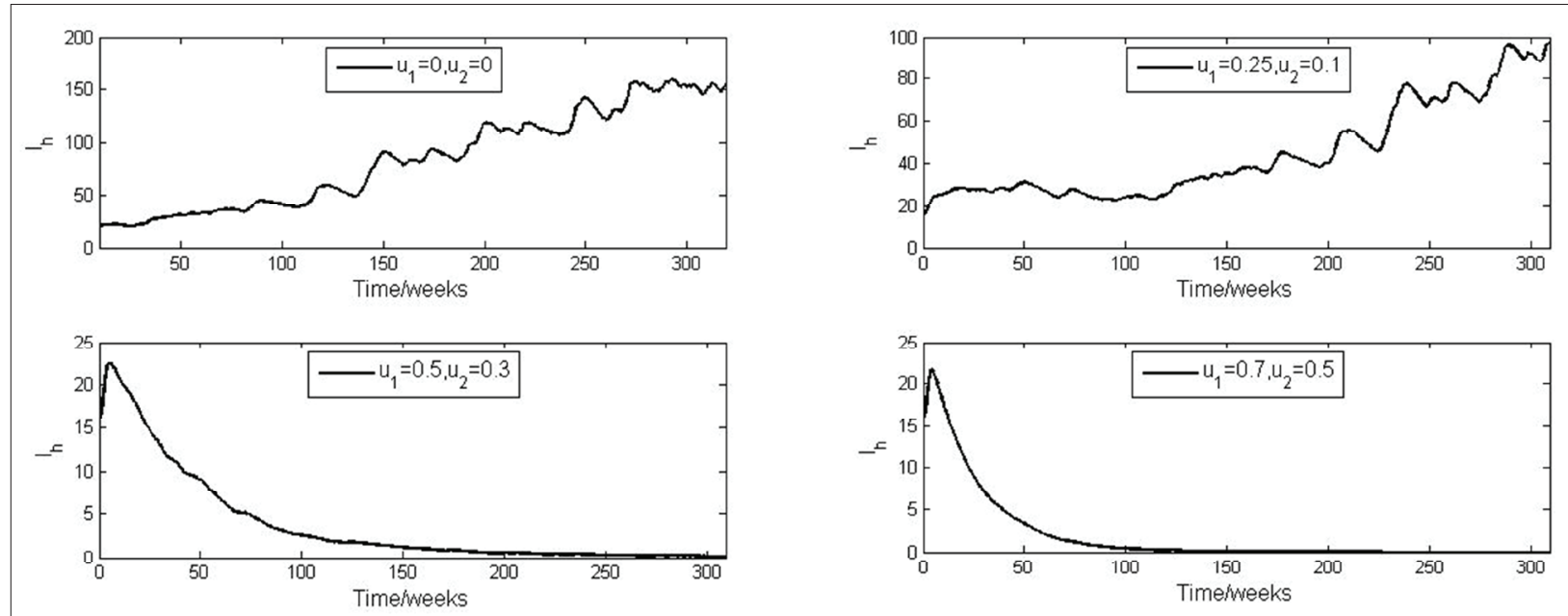

Figure 7: Simulated infected human population distribution only with the rainfall variation (fixed temperature effect) for various combinations of constant continuous control measures from year 2006 - 2012 in Colombo. Here, $u_{1}=0$ and $u_{2}=0$ (top-left); $u_{1}=0.25$ and $u_{2}=0.1$ (top-right); $u_{1}=0.5$ and $u_{2}=0.3$ (bottom-left); $u_{1}=0.5$ and $u_{2}=0.7$ (bottom-right) 
that the effective constant continuous control strategies have a negative impact on the dengue mosquitoes. This mosquito density dynamic is used to investigate the behaviour of the infected human distribution given the rainfall variation and control measures and the results are shown in Figure 7. The results indicate that the dynamics of the infected humans have changed and the number of infections have reduced once the control strategies are implemented. The results in Figure 7 suggests that a high level of efficient control measures can be used to eradicate the dengue infections in urban Colombo.

\section{CONCLUSION}

A fuzzy based mathematical model was developed to analyse mosquito density considering only the climate variation. For further improvement, other external factors favourable for mosquitoes can also be included. Optimal control techniques can be used to investigate the point in time where the control measures should be introduced to minimise the magnitude of the disease outbreak.

\section{REFERENCES}

1. Allis A.M., Garcia A.J. \& Morrison A.C. (2011). Parameterization and sensitivity analysis of a complex simulation model for mosquito population dynamics, dengue transmission, and their control. The American Society of Tropical Medicine and Hygiene 85: 257 - 264.

2. Bhatia R., Dash A.P. \& Sunyoto T. (2013). Changing epidemiology of dengue in South-East Asia. WHO SouthEast Asia Journal of Public Health 2(1): 23 - 27.

3. Cartwright J. \& Piro O. (1992). The dynamics of RungeKutta methods. International Journal of Bifurcation and Chaos 2: $427-449$.

4. Chaves L., Morrison A.C., Kirtron U.D. \& Scott T.W. (2011). Nonlinear impacts of climatic variability on the density dependent regulation of an insect vector of disease. Global Change Biology 18: 457 - 468.

5. Epidemiology Unit, Ministry of Health (2005). National Plan of Action for Prevention and Control of Dengue Fever 2005 - 2009. Epidemiology Unit, Ministry of Health, Sri Lanka.

6. Epidemiology Unit, Ministry of Health, Sri Lanka, http://www.epid.gov.lk/web/index.php? option $=$ com content\&view $=$ article\&id $=141 \&$ Itemid $=465 \&$ lang $=e n$, Accessed 4 July 2016.

7. Estevaa L. \& Vargas C. (1998). Analysis of a dengue disease transmission model. Mathematical Biosciences 150: $131-151$.

8. Franke J. \& Yakubu A. (2005). Population models with periodic recruitment functions and survival rates. Journal of Difference Equations and Applications 11(14):
$1169-1184$

9. Huang X., Clements C.A., Williams G., Milinovich G. \& $\mathrm{Hu}$ W. (2013). A threshold analysis of dengue transmission in terms of weather variables and imported dengue cases in Australia. Emerging Microbes and Infections 2(12): e87. DOI: https://doi.org/10.1038/emi.2013.85

10. Lloyd L.S. (2003). Best Practices for Dengue Prevention and Control in the Americas. Environmental Health Project, U.S. Agency for International Development, Washington, USA.

11. Ma S. \& Xia Y. (2009). Mathematical Understanding of Infectious Disease Dynamics. Lecture Notes Series, volume 16. Institute for Mathematical Sciences, National University of Singapore, Singapore.

12. Medeiros L.C., Castilho C.A.R. \& Braga C. (2011). Modelling the dynamic transmission of dengue fever: investigating disease persistence. Plos Neglected Tropical Diseases 5(2): 1 - 14.

13. Murray E.A., Quam M. \& Smith A.W. (2013). Epidemiology of dengue: past, present and future prospects. Clinical Epidemiology 5: 299 - 309.

14. Naish S., Dale P., Mackenzie J.S., McBride J., Mengersen K. \& Tong S. (2014). Climate change and dengue: a critical and systematic review of quantitative modeling approaches. BMC Infectious Diseases 14: 167. DOI: https://doi.org/10.1186/1471-2334-14-167

15. Parks W. \& Lloyd L. (2004). Planning Social Mobilization and Communication for Dengue Fever Prevention and Control: a Step-by-Step Guide, p. 138. World Health Organisation, Geneva, Switzerland.

16. Pongsumpun P. (2006). Transmission model for dengue disease with and without the effect of extrinsic incubation period. KMITL Science and Technology Journal 6(2): $74-82$.

17. Racloz V., Ramsey R., Tong S. \& Hu W. (2012). Surveillance of dengue fever virus: a review of epidemiological models and early warning systems. Plos Neglected Tropical Diseases 6(5): 1 - 9.

18. Suli E. (2014). Numerical Solution of Ordinary Differential Equations, pp. 13 - 19. Mathematical Institute, University of Oxford, Oxford, UK.

19. Thai D. (2012). Dengue: a trilogy of people, mosquitoes and the virus. Current epidemiology and pathogenesis in (non-)endemic settings. Thesis, Faculty of Medicine, Oxford Clinical Research Unit (OUCRU), Ho Chi Minh City, Vietnam.

20. Thalagala N. (2012). Health System Cost for Dengue Control and Management in Colombo District, Sri Lanka in 2012. Dengue Tool Surveillance Project, Epidemiology Unit, Ministry of Health, Sri Lanka.

21. Turchin P. (2003). Complex population dynamics. Monographs in Population Biology, pp. 19 - 98. Princeton University Press, USA.

22. Zimmermann H. (2010). Fuzzy set theory. Wiley Interdisciplinary Reviews: Computational Statistics 2(3): $371-332$. 